\title{
Eletrostática em sistemas coloidais carregados
}

\author{
Electrostatics in charged colloidal systems
}

\author{
Igor Rochaid Oliveira Ramos ${ }^{*}$, João Philipe Macedo Braga ${ }^{3}$, João Vitor Alencar Ataíde ${ }^{1}$, \\ Alexsandro Pereira Lima ${ }^{4}$, Lino Holanda \\ ${ }^{1}$ Universidade Federal Rural do Semi-Árido, Campus Pau dos Ferros, Pau dos Ferros, RN, Brasil \\ ${ }^{2}$ Universidade Estadual Vale do Acaraú, Campus Cidao, Sobral, CE, Brasil \\ ${ }^{3}$ Universidade da Integração Internacional da Lusofonia Afro-Brasileira, Campus dos Palmares, Acarape, CE, Brasil \\ ${ }^{4}$ Universidade Federal do Rio Grande do Norte, Escola de Ciência e Tecnologia, Natal, RN, Brasil
}

Recebido em 19 de Fevereiro de 2018. Revisado em 07 de Maio de 2018. Aceito em 09 de Maio de 2018.

\begin{abstract}
Neste trabalho apresentamos um estudo da eletrostática aplicada aos sistemas coloidais cujo intuito é fornecer aos alunos de graduação e pós-graduação em Física um material didático complementar que possibilite uma visão mais ampla dos conceitos estudados em sala através de uma aplicação direta da teoria. Para tanto, utilizamos um modelo de um sistema coloidal a partir do qual derivamos as expressões para a Força, Campo e Potencial elétricos, tanto de distribuições discretas de carga como para distribuições contínuas. Encontramos ainda equações equivalentes para a Lei de Gauss, tanto na forma integral como na forma diferencial.
\end{abstract}

Palavras-chave: Eletrostática, Colóides

In this work we present a study of the electrostatics applied to colloidal systems whose purpose is to provide undergraduate and postgraduate students in Physics with a broader view of the concepts studied in the classroom by direct application of the theory. To do so, we used a model of a colloidal system from which we derive the expressions for the Electric Force, Field and Potential, both for discrete and continuous charge distributions. We also find equivalent equations for the Gauss's Law, both in integral and differential forms.

Keywords: Electrostatic, Colloids

\section{Introdução}

A teoria do eletromagnetismo fora proposta por Maxwell no final do século XIX, sendo considerada uma das teorias mais bem consolidadas da Física. Todavia, o estudo dessa disciplina é feito até hoje de forma separada, primeiro os alunos são apresentados à eletricidade e em seguida ao magnetismo. E dentro dos conceitos elétricos, os estudantes aprendem primeiro o conteúdo relativo à eletrostática.

No estudo da eletrostática, consideramos que todas as cargas fontes são estacionárias, e que a carga de prova pode estar ou não em movimento. Partimos, portanto, de dois fatos experimentais, a saber: a lei de Coulomb e o princípio da superposição. A lei de Coulomb nos diz que o módulo da força elétrica entre duas cargas pontuais é proporcional ao produto das cargas e inversamente proporcional ao quadrado da distância que as separa e o princípio da superposição, por sua vez, afirma que a interação entre duas cargas quaisquer não é modificada pela presença de outras cargas [1] 6].

A partir dessas considerações define-se, então, o campo elétrico devido a uma dada distribuição de cargas. Uma vez calculado o campo elétrico, podemos encontrar a força

*Endereço de correspondência: holandaj@ifi.unicamp.br que atua sobre uma determinada carga de prova que seja colocada nas proximidades da distribuição. Em princípio, podemos dizer que o estudo da eletrostática resume-se aos fenômenos descritos acima, o que, de certa maneira, é bem verdade, pois os passos posteriores resumem-se a encontrar ferramentas matemáticas para facilitar o cálculo do campo elétrico (como o potencial elétrico e a equação de Poisson) e, consequentemente, da força elétrica.

Didaticamente falando, a maioria dos livros adotados na graduação e pós-graduação apresentam a eletrostática seguindo basicamente a mesma sequência: introdução histórica e formalismo matemático. Ao longo desse processo, os alunos são apresentados aos conceitos de Carga, Força e Campo tanto para distribuições discretas como para distribuições contínuas de carga (e. g. Refs [2] 3 ), seguido de uma aplicação em meios dielétricos (e. g. Refs 3 5]). Neste trabalho, apresentamos o formalismo matemático da eletrostática aplicado aos sistemas coloidais. Acreditamos que a discussão da eletrostática do ponto de vista de uma aplicação possa fornecer ao aluno uma maior compreensão do conteúdo ministrado e, consequentemente, uma melhor fixação do assunto. Nesse sentido, não pretendemos aqui apresentar novos resultados sobre a eletrostática ou mesmo sobre sistemas coloidais, mas 
reunir o conhecimento existente na literatura sobre os colóides em um único local aplicando-os no contexto da eletrostática, de tal modo que possa ser utilizado como material complementar nas disciplinas de eletromagmetismo nos cursos de graduação e pós-graduação em física.

\subsection{Sistemas coloidais}

O termo sistema coloidal ou suspensão coloidal é frequentemente usado quando lidamos com materiais que são compostos de partículas de tamanhos típicos variando entre $1 \mathrm{~nm}$ e $1 \mu \mathrm{m}$ (partículas mesoscópicas) dispersas em um solvente cujas moléculas são muito menores em tamanho. As partículas mesoscópicas formam a fase dispersa e o solvente, a fase dispersante. Quando as partículas dispersas têm tamanhos diferentes, o sistema coloidal é denominado polidisperso. Por outro lado, os sistemas coloidais com partículas de mesmo tamanho são denominados monodispersos [7].

Esse tipo de sistema tem atraído a atenção de muitos pesquisadores nas últimas décadas. As principais razões da importância do sistema de colóides são: 1) ao contrário dos sistemas atômicos, nos quais as interações entre as partículas são determinadas pela sua estrutura eletrônica e, portanto, não podem ser controladas externamente 8, 9], as interações entre partículas coloidais e, consequentemente, as propriedades físico-químicas do sistema, podem ser modificadas externamente controlando, por exemplo, a temperatura, a concentração de eletrólitos (sais, ácidos ou bases, por exemplo), a composição das partículas dispersas, o tipo de solvente empregado e/ou um campo magnético externo, dependendo do tipo de partícula no sistema; 2) do ponto de vista experimental, o tamanho das partículas coloidais é da ordem de grandeza do comprimento de onda da luz visível (400 $\mathrm{nm}$ $700 \mathrm{~nm}$ ), tornando possível estudar este sistema através de experimentos envolvendo espalhamento de luz [8,9]. Além disso, o movimento das partículas pode ser observado diretamente usando vídeo-microscopia e o estado do sistema analisado em tempo real $10-13]$.

\subsection{Sistemas coloidais magnéticos}

Fenômenos interessantes ocorrem ao estudarmos sistemas magnéticos coloidais, os quais são constituídos de uma fase dispersa sólida composta de nanopartículas magnéticas distribuídas em um meio dispersante líquido. Tal sistema magnético coloidal é designado por ferrofluido ou fluido magnético. As nanopartículas são mono-domínios magnéticos, usualmente esféricas, com diâmetro abaixo de $15 \mathrm{~nm}$ (diâmetro crítico) 14. Para entender como surgem os mono-domínios magnéticos usaremos o conceito de sólidos ferromagnéticos. Sólidos ferromagnéticos são materiais não-lineares (possuem magnetização mesmo na ausência de campo magnético externo) compostos por regiões onde os dipolos magnéticos dos átomos individuais são orientados numa direção preferencial; essas regiões são denominadas domínios magnéticos 3, 15.
Como exemplos de materiais ferromagnéticos temos o ferro, níquel, cobalto e algumas de suas ligas e compostos 3, 15. O dipolo magnético é responsável pelas características magnéticas do material e tem sua origem no momento angular total $\vec{J}$, o qual é o resultado do acoplamento entre os momentos angulares orbital e de spin dos elétrons, $\vec{J}=\vec{L}+\vec{S}$. Quando o tamanho do material ferromagnético é reduzido, os domínios se fundem gerando uma partícula de mono-domínio magnético. Este tamanho depende do material e é denominado tamanho crítico, no entanto, caso a partícula gerada seja esférica, denomina-se diâmetro crítico 14, 16, 17.

Em 1946, Kittel [14 apresentou a primeira estimativa do valor do diâmetro crítico a partir do qual a partícula esférica é dita mono-domínio. Esse diâmetro crítico é de, aproximadamente, $15 \mathrm{~nm}$ [14,18, 19]. As partículas magnéticas com diâmetro menor do que o diâmetro crítico, apresentam comportamento superparamagnético, devido ao fato de o momento magnético total estar entre o paramagnético e o ferromagnético 19,20]. Com isso, a magnetização de saturação é maior nos materiais superparamagnéticos do que nos paramagnéticos. Outras características dos materiais superparamagneticos são a ausência de histerese e campo coercivo (campo magnético necessário para remover a magnetização residual) nulo [19].

\subsection{Estabilidade de sistemas coloidais}

Uma característica fundamental dos sistemas coloidais é a tendência natural de aglomeração das nanopartículas e, consequentemente, a coagulação 20]. Para minimizar a possibilidade de aglomeração, as partículas dispersas devem ser grandes o bastante para que o solvente possa ser descrito como um meio contínuo e homogêneo e pequenas o suficiente de modo a apresentar movimento browniano. Além disso, as partículas podem ser revestidas com uma camada de determinadas substâncias, que são escolhidas de acordo com o fim desejado 21]. Dependendo do tipo de revestimento escolhido para se obter a estabilidade dos fluidos magnéticos, estes podem ser classificados em surfactados ou iônicos.

Nos fluidos magnéticos surfactados, a superfície da nanopartícula é recoberta por uma camada molecular chamada surfactante, com espessura da ordem de 1 a $3 \mathrm{~nm}$, que adere à superfície da partícula criando uma repulsão entre elas, impedindo assim a aglomeração e promovendo a estabilidade do fluido 15,18 .

Nos fluidos magnéticos iônicos, a estabilidade coloidal está associada à repulsão eletrostática. O solvente geralmente é a água, e a nanopartícula é carregada com uma densidade superficial de carga elétrica, que depende do $\mathrm{pH}$ do meio: positiva em meio ácido, negativa em meio básico e nula em região de neutralidade. O processo de carregamento envolve reações ácido-base de Brönsted, nas quais, grupos superficiais são protonados ou desprotonados 22,23. Outros solventes polares como o 
dimetilsulfóxido (DMSO) também têm sido empregados na elaboração de fluidos magnéticos iônicos 24]. Dessa forma, nos fluidos magnéticos surfactados e iônicos, as nanopartículas se mantêm em suspensão na forma de objetos isolados, evitando assim a aglomeração e subsequente precipitação (estabilidade coloidal).

\subsection{Sistemas coloidais carregados}

As partículas coloidais adquirem cargas elétricas na superfície, quando expostas ao contato com solvente polar, por diferentes mecanismos, tais como dissociação de grupos da superfície e adsorção ou dissolução de íons da superfície. A carga da superfície da partícula influencia a distribuição dos íons (contra-íons e co-íons) da solução na sua vizinhança através da interação coulombiana atrativa ou repulsiva entre as cargas 7]. Como consequência, o potencial entre a superfície da partícula e o interior da solução, diminui mais rapidamente devido à dupla camada de cargas que se forma ao redor da partícula 7].

Num sistema de partículas coloidais carregadas dispersas em um meio contendo íons, a interação real entre essas partículas coloidais é dada pelo potencial de $\mathrm{Yu}-$ kawa ou potencial de Debye-Hückel [25 27]. Neste caso, para uma determinada temperatura, a interação entre as partículas coloidais é blindada pela nuvem iônica que envolve as partículas e o alcance da interação pode ser modificado alterando a concentração de eletrólito 25.26 . Para uma baixa concentração de íons, a interação pode ser aproximada por um potencial coulombiano. Por outro lado, em um sistema coloidal eletricamente estabilizado, a carga dos colóides pode ser controlada pelo pH da solução ou pela adição de sais quando é possível a adsorção específica 28 30].

No estudo da eletrostática em sistemas coloidais carregados, embora possamos utilizar o princípio da superposição, não partiremos de uma lei experimental análoga à lei de Coulomb. Em vez disso, calcularemos primeiro o potencial elétrico devido a uma carga pontual em uma dada distância, e a partir do potencial, calcularemos o campo elétrico e, finalmente, a força elétrica entre duas cargas.

\section{Modelo}

Os sistemas coloidais, constituídos por uma fase dispersa (partículas coloidais) e pela fase contínua (solvente), podem ocorrer em oito classes de colóides (dependendo do estado físico no qual a matéria se encontra) de acordo com o quadro da figura 1.

Neste trabalho, analisaremos um sistema coloidal do tipo Sol, constituído de partículas sólidas finamente divididas dispersas em um meio dispersante líquido (Figura 2). Nesse tipo de colóide, as partículas estão bem separadas e o sistema como um todo apresenta aspecto líquido. Alguns exemplos desse tipo de sistema aparecem quando misturamos gelatina em água ou goma arábica em água.

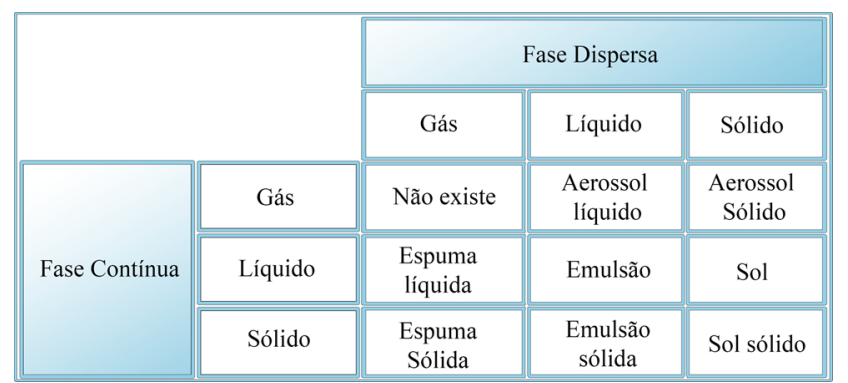

Figura 1: Classificação do sistema coloidal em termos do estado físico da matéria. a)

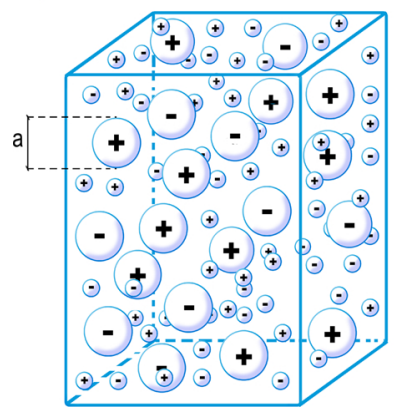

b)

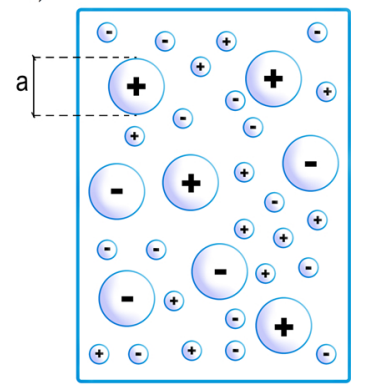

Figura 2: Sistema coloidal formado por partículas esféricas carregadas positivamente e negativamente dispersas em um volume $\tau$, na presença de partículas pontuais com cargas positivas e negativas (figura $2 \mathrm{a}$ ). A figura $2 \mathrm{~b}$ mostra uma visão lateral do sistema evidenciando uma de suas faces para melhor visualização.

No entanto, para conseguirmos uma estabilização do sistema, precisamos de algo para evitar a coagulação das partículas coloidais que, no nosso caso, é conseguido através de cargas elétricas formadas na superfície das partículas.

Nesse sentido, consideramos um modelo teórico que consiste em um sistema composto de muitas partículas coloidais esféricas de diâmetro $a$, que possuem cargas positivas ou negativas de módulo $Q$ em seus centros, na presença de partículas carregadas pontuais positivas ou negativas, com carga de módulo $q$ (representando os contra-íons e os co-íons), dispersas em um meio contínuo de permissividade $\epsilon$, como mostra a Figura 2. Além disso, o sistema como um todo é eletricamente neutro, ou seja, a quantidade de partículas com carga positiva é igual à quantidade de partículas com carga negativa.

\subsection{Cálculo do potencial elétrico}

De acordo com o modelo apresentado acima, vamos considerar uma partícula coloidal $i$, com carga positiva, situada em $r=0$. Dessa forma, o potencial elétrico $V$ gerado por essa partícula a uma distância $r$, na presença dos contra-íons e dos co-íons, dentro do volume $\tau$, pode ser encontrado através da equação de Poisson. Assim, consideraremos duas regiões: a região I $(r>a)$ e a região II $(0<r \leq a)$. Na região I, temos 


$$
\nabla^{2} V=-\frac{\rho_{q}(r)}{\epsilon}
$$

onde $\rho_{q}(r)$ é a densidade volumétrica de cargas dos íons (contra-íons e co-íons) e $\epsilon$ é a permissividade do meio. Por outro lado, na região II, temos $\rho_{q}=0$ e, portanto, a equação de Poisson reduz-se à equação de Laplace, ou seja

$$
\nabla^{2} V=0
$$

A distribuição dos íons no meio varia no espaço devido às interações eletrostáticas com a partícula coloidal fixa $i$. Para levar em conta essas interações, utilizaremos a distribuição de Boltzmann envolvendo a energia de interação entre os íons e a partícula fixa $i$. Assim, podemos escrever

$$
\rho_{q}(r)=q \rho_{+} e^{-\beta W_{++}(r)}-q \rho_{-} e^{-\beta W_{+-}(r)}
$$

onde $\beta=1 / k_{B} T$ ( $k_{B}$ é a constante de Boltzmann e $T$ é a temperatura do sistema), $\rho_{+}$e $\rho_{-}$são a densidade de co-íons e de contra-íons no meio (não devemos pensar que se trata de uma densidade volumétrica de cargas e, sim, de uma densidade de partículas: número de partículas (co-íons ou contra-íons) por unidade de volume), respectivamente; $W_{i j}$ é o trabalho necessário para trazer os íons do infinito e colocá-los a uma distância $r$ da partícula coloidal $i[26$. Esse trabalho pode ser escrito como

$$
W_{i j}(r)=q_{j} V(r)
$$

onde $q_{j}$ é a carga do íon $j$ e $V(r)$ é o potencial eletrostático a uma distância $r$ devido à partícula coloidal $i$ na origem. Assim, a equação de Poisson pode ser escrita como

$$
\nabla^{2} V=-\frac{1}{\epsilon}\left(q \rho_{+} e^{-\beta q V(r)}-q \rho_{-} e^{\beta q V(r)}\right)
$$

Além disso, o sistema é eletricamente neutro, ou seja, $\rho_{+}=\rho_{-}=\rho / 2$, o que nos permite reescrever a Equação (5) da seguinte forma

$$
\nabla^{2} V=\frac{\rho q}{\epsilon} \sinh (\beta q V)
$$

a qual é uma equação diferencial de segunda ordem nãolinear, conhecida como equação de Poisson-Boltzmann. Para linearizarmos esta equação, vamos supor que a energia térmica é muito maior que a energia potencial elétrica de interação entre as partículas. Isso equivale a escrever $\beta q V \ll 1 \mathrm{e}$, portanto, a Equação (6) reduz-se à equação de Helmholtz 26

$$
\nabla^{2} V=\kappa^{2} V
$$

onde

$$
\kappa=\sqrt{\frac{q^{2} \rho}{k_{B} T \epsilon}}
$$

cujo significado físico será discutido posteriormente.
Como o problema tem simetria esférica, precisamos encontrar somente a solução para a parte radial da equação de Helmholtz, a qual pode ser escrita, em coordenadas esféricas, como

$$
\frac{1}{r^{2}} \frac{d}{d r}\left(r^{2} \frac{d V}{d r}\right)=\kappa^{2} V
$$

A solução desta equação pode ser encontrada fazendo uma simples mudança de variável da forma $u=r V(r)$. O resultado encontrado será

$$
V=A \frac{e^{\kappa r}}{r}+B \frac{e^{-\kappa r}}{r}
$$

O valor da constante A pode ser facilmente determinado através da condição de que o potencial é nulo para grandes distâncias. Utilizando essa condição, encontramos $A=0$. Assim, ficamos com

$$
V=B \frac{e^{-\kappa r}}{r}
$$

Para a região II, devemos resolver a equação de Laplace

$$
\nabla^{2} V=0
$$

Utilizando novamente a parte radial do Laplaciano em coordenadas esféricas e a mesma mudança de variável, obtemos

$$
V=C+\frac{D}{r}
$$

Como a dependência com a distância do segundo termo da Equação (13) é do tipo 1/r, podemos concluir que ele está relacionado ao potencial produzido pela partícula coloidal fixa $i$ de carga $Q$. Consequentemente, temos que

$$
D=\frac{Q}{4 \pi \epsilon}
$$

Para encontrar as constantes $B$ e $C$, utilizaremos a continuidade do potencial e do campo elétrico em $r=a$ (na fronteira que separa as regiões I e II) 26. Fazendo isso, obtemos

$$
B \frac{e^{-\kappa a}}{a}=C+\frac{D}{a}
$$

e

$$
\begin{gathered}
\frac{D}{a^{2}}=B\left(\frac{e^{-\kappa a}}{a^{2}}+\kappa \frac{e^{-\kappa a}}{a}\right) \\
D=B e^{-\kappa a}(1+\kappa a)
\end{gathered}
$$

e, com isso, $B$ e $C$ são dados por

$$
\begin{gathered}
B=\frac{Q}{4 \pi \epsilon} \frac{e^{\kappa a}}{(1+\kappa a)} \\
C=-\frac{Q}{4 \pi \epsilon} \frac{\kappa}{(1+\kappa a)}
\end{gathered}
$$

Dessa forma, o potencial eletrostático para $r \leq a$ é 


$$
V_{<}(r)=\frac{Q}{4 \pi \epsilon r}-\frac{Q \kappa}{4 \pi \epsilon(1+\kappa a)}
$$

enquanto para $r>a$,

$$
V_{>}(r)=\frac{Q e^{\kappa a}}{4 \pi \epsilon(1+\kappa a)} \frac{e^{-\kappa r}}{r}
$$

A Equação 20 mostra que o potencial eletrostático para $r \leq a$ é gerado tanto pela partícula coloidal $i$ como pela nuvem iônica que envolve a partícula. Por outro lado, para $r>a$, a Equação (21) nos permite concluir que o potencial eletrostático gerado pela partícula coloidal é blindado exponencialmente pela nuvem iônica que a envolve. O alcance do potencial está diretamente relacionado ao parâmetro $\kappa$, sendo $l=\kappa^{-1}$ a distância na qual a interação eletrostática permanece significativa, ou seja, $l=\kappa^{-1}$ representa a espessura da dupla camada de cargas que circunda a partícula coloidal, o qual depende da carga, da densidade dos íons e da temperatura da solução. Para um sistema com temperatura constante, o alcance do potencial pode ser reduzido aumentando o valor da carga do íon (para uma dada densidade de íons) ou aumentando a densidade de íons do meio (para um dado valor de carga). De outro modo, quanto menor a temperatura da solução, menor será a probabilidade dos íons superarem a atração eletrostática, aumentando assim a blindagem da partícula coloidal e, consequentemente, menor será o alcance do potencial.

Podemos demonstrar também que o potencial de Coulomb é um caso particular para baixas densidades de íons da solução. Para encontrar o potencial eletrostático gerado por uma carga pontual $Q$ num ponto situado a uma distância $r$ da carga, fazemos o diâmetro $a$ da partícula esférica igual a zero na Equação 21 e, como resultado, obtemos

$$
V(r)=\frac{Q}{4 \pi \epsilon} \frac{e^{-\kappa r}}{r}
$$

Note que se a partícula encontra-se no vácuo, $\epsilon=\epsilon_{0}$ ( $\epsilon_{0}$ é a permissividade do vácuo) e $\kappa=0$; com isso encontramos

$$
V(r)=\frac{1}{4 \pi \epsilon_{0}} \frac{Q}{r}
$$

que é o potencial eletrostático coulombiano devido a uma carga pontual (q. e. d.).

A Equação (22) nos fornece o potencial elétrico efetivamente gerado por uma partícula carregada nesse modelo de sistema coloidal. Portanto, conforme mostraremos nas próximas seções, nesse tipo de sistema tudo se passa como se a lei fundamental de interação entre partículas carregadas não fosse mais a lei de Coulomb, mas uma lei de interação de alcance muito menor devido ao efeito de blindagem pela nuvem iônica que circunda a partícula medido pelo parâmetro $\kappa$. Nesse sentido, podemos considerar que, efetivamente, a eletrostática em sistemas coloidais carregados se resume a essa nova lei de interação somada ao princípio da superposição.

\section{Campo elétrico gerado por uma carga pontual}

Continuando o desenvolvimento da eletrostática a partir do modelo que estamos considerando, iremos encontrar o campo elétrico gerado por uma carga pontual. A relação entre o campo elétrico $\vec{E}$ e o potencial $V$ é dada por

$$
\vec{E}=-\vec{\nabla} V
$$

onde, da Equação 22 podemos escrever

$$
\vec{E}=-\frac{Q}{4 \pi \epsilon} \frac{\partial}{\partial r}\left(\frac{e^{-\kappa r}}{r}\right) \hat{r}
$$

o que nos permite encontrar a expressão para o campo elétrico

$$
\vec{E}=\frac{Q(1+\kappa r)}{4 \pi \epsilon} \frac{e^{-\kappa r}}{r^{2}} \hat{r}
$$

\section{Força elétrica entre duas cargas pontuais}

Agora, de posse do campo elétrico, podemos encontrar uma expressão para a força de interação eletrostática que atua entre duas cargas pontuais.

Sabemos que a força elétrica que atua em uma carga de prova $q_{0}$ na presença de um campo elétrico gerado pela carga $Q$ é dada por

$$
\vec{F}=q_{0} \vec{E}
$$

Usando a Equação 26, encontramos a expressão para a força elétrica como sendo

$$
\vec{F}=\frac{1}{4 \pi \epsilon} \frac{q_{0} Q}{r^{2}}(1+\kappa r) e^{-\kappa r} \hat{r}
$$

A Equação (28) substitui efetivamente a Lei de Coulomb nesse nosso modelo de sistemas coloidais carregados, tendo a mesma como caso particular $(\kappa=0)$.

\section{Campo elétrico gerado por uma distribuição discreta de cargas pontuais}

Dando continuidade ao nosso estudo da eletrostática aplicada a sistemas coloidais, iremos analisar a situação de um campo elétrico gerado por uma distribuição discreta de cargas. Para tanto, iremos considerar $N$ cargas pontuais $Q_{1}, Q_{2}, Q_{3}, \ldots, Q_{N}$, situadas às distâncias $r_{1}, r_{2}$, $r_{3}, \ldots, r_{N}$, respectivamente, de um ponto do espaço.

Podemos encontrar o campo elétrico gerado por essas cargas, nesse ponto, a partir da generalização da Equação (26), o que nos dá

$$
\vec{E}(\vec{r})=\frac{1}{4 \pi \epsilon} \sum_{i}^{N} \frac{Q_{i}\left(1+\kappa r_{i}\right) e^{-\kappa r_{i}}}{r_{i}^{2}} \hat{r}_{i}
$$




\section{Campo elétrico gerado por uma distribuição contínua de cargas}

Se ao invés de $N$ cargas pontuais discretas, tivermos uma distribuição contínua de cargas sobre uma dada região, o somatório na Equação (29) será substituído por uma integral. Com isso, podemos escrever a expressão para o campo elétrico da forma

$$
\vec{E}(\vec{r})=\frac{1}{4 \pi \epsilon} \int \frac{e^{-\kappa r}(1+\kappa r) \hat{r}}{r^{2}} d Q
$$

onde $r$ é a distância entre $d Q$ e o ponto onde queremos calcular o campo.

\section{Lei de Gauss na forma integral}

O fluxo do campo elétrico gerado por uma carga pontual na origem através de uma superfície esférica de raio $R$, é dado por

$$
\oint_{S} \vec{E} \cdot d \vec{S}
$$

Substituindo a expressão encontrada para o campo elétrico, dado pela Equação 26 e fazendo $r \equiv R$, podemos obter a seguinte expressão

$$
\oint_{S} \vec{E} \cdot d \vec{S}=\oint_{S} \frac{Q(1+\kappa R)}{4 \pi \epsilon} \frac{e^{-\kappa R}}{R^{2}} d S \hat{r} \cdot \hat{r}
$$

de modo que,

$$
\oint_{S} \vec{E} \cdot d \vec{S}=\frac{Q}{\epsilon}(1+\kappa R) e^{-\kappa R}
$$

Por outro lado, a integral de volume

$$
\int_{\Omega} V(r) d \Omega
$$

onde $d \Omega=4 \pi r^{2} d r$ é o elemento de volume em coordenadas esféricas, e $V(r)$ é o potencial elétrico dado pela Equação 22, nos dá

$$
\int_{\Omega} V(r) d \Omega=\frac{Q}{\epsilon \kappa^{2}}\left[1-(1+\kappa R) e^{-\kappa R}\right]
$$

Utilizando as Equações (33) e (35), obtemos a seguinte expressão

$$
\oint_{S} \vec{E} \cdot d \vec{S}+\kappa^{2} \int_{\Omega} V(r) d \Omega=\frac{Q}{\epsilon}
$$

Agora, se em vez de uma carga na origem tivermos $N$ cargas distribuídas em torno da origem, pelo princípio da superposição, o campo elétrico total será a soma vetorial de todos os campos individuais $1-6$. Com isso,

$$
\vec{E}=\sum_{i=1}^{N} \vec{E}_{i}
$$

$$
V=\sum_{i=1}^{N} V_{i}
$$

tal que

$$
\begin{gathered}
\oint_{S} \vec{E} \cdot d \vec{S}+\kappa^{2} \int_{\Omega} V(r) d \Omega= \\
\sum_{i=1}^{N}\left(\oint_{S} \vec{E}_{i} \cdot d \vec{S}+\kappa^{2} \int_{\Omega} V_{i}(r) d \Omega\right) \\
\oint_{S} \vec{E} \cdot d \vec{S}+\kappa^{2} \int_{\Omega} V(r) d \Omega=\sum_{i=1}^{N} \frac{Q_{i}}{\epsilon}
\end{gathered}
$$

Logo, a nova lei de Gauss na forma integral é dada por

$$
\oint_{S} \vec{E} \cdot d \vec{S}+\kappa^{2} \int_{\Omega} V(r) d \Omega=\frac{Q_{e n v}}{\epsilon}
$$

onde $Q_{e n v}$ é a carga total envolvida pela superfície S.

\section{Lei de Gauss na forma diferencial e equação de Poisson}

Para obter a lei de Gauss na forma diferencial, usaremos o teorema de Gauss dado por

$$
\oint_{S} \vec{v} \cdot d \vec{S}=\int_{\Omega}(\vec{\nabla} \cdot \vec{v}) d \Omega
$$

onde $\vec{v}$ é um campo vetorial. Além disso, sabemos que a carga envolvida pela superfície é dada por

$$
Q_{e n v}=\int_{\Omega} \rho_{Q} d \Omega
$$

Utilizando estes resultados e a lei de Gauss na forma integral, obtemos

$$
\vec{\nabla} \cdot \vec{E}+\kappa^{2} V(r)=\frac{\rho_{Q}}{\epsilon}
$$

que é a lei de Gauss na forma diferencial.

Agora, usando $\vec{E}=-\vec{\nabla} V$, obtemos uma equação análoga a equação de Poisson para a eletrostática em sistemas coloidais carregados

$$
-\nabla^{2} V+\kappa^{2} V(r)=\frac{\rho_{Q}}{\epsilon}
$$

\section{Conclusão}

Os sistemas coloidais apresentam uma grande gama de aplicação na indústria (e. g. maionese, creme dental e xampu) como também existem de várias formas na natureza (e. g. neblina, fumaça, pedra-pomes e pérola). Neste trabalho, apresentamos um estudo da eletrostática aplicada a um modelo de sistema coloidal, onde derivamos as equações para a Força, Campo e Potencial elétricos. Desenvolvemos também expressões equivalentes para a 
Lei de Gauss tanto em sua forma integral como na forma diferencial. Tal estudo proporcionará ao estudante uma melhor compreensão dos conceitos da eletrostática pelo fato da aplicação direta de seu conhecimento, constituindo um interessante material complementar para as disciplinas de eletromagnetismo nos cursos de graduação e pós-graduação em física.

\section{Referências}

[1] D. Halliday, R. Resnick e J. Walker, Fundamentos de Física (LTC editora, Rio de Janeiro, 2012), v.3, $9^{a}$ ed.

[2] H.M. Nussenzveig, Curso de Física Básica (Edgard Blücher, São Paulo, 1997), v. 3, $1^{a}$ ed.

[3] D.J. Griffiths, Introduction to Electrodynamics (Prentice Hall, New Jersey, 1999), $3^{a}$ ed.

[4] J.R. Reitz, F.J. Milford e R.W. Chisty, Fundamentos da Teoria Eletromagnética (Editora Campus, Rio de Janeiro, 1982), $1^{a}$ ed.

[5] J.D. Jackson, Classical Electrodynamics (John Wiley \& Sons, New Jersey 1999).

[6] E.M. Purcell, Curso de Fisica de Berkeley: Eletricidade e Magnetismo (Edgard Blücher, São Paulo, 1973), v. 2.

[7] M. Jafelicci Junior e L.C. Varanda, QNEsc 9, 9 (1999).

[8] C.N. Likos, Physics Reports 348, 267 (2001).

[9] P. Keim, G. Maret, U. Herz e H.H. von Grünberg, Phys. Rev. Lett. 92, 215504 (2004).

[10] F.M. Peeters e W. Xiaoguang, Phys. Rev. A. 35, 3109 (1987).

[11] F. Ebert, P. Dillmann, G. Maret e P. Keim, Rev. Sci. Instrum. 80, 083902 (2009).

[12] A.D. Law, D. Martin, A. Buzza e T.S. Horozov, Phys. Rev. Lett. 106, 128302 (2011).

[13] I.R.O. Ramos, W.P. Ferreira, F.F. Munarin e F.M. Peeters, Phys. Rev. E. 90, 062311 (2014).

[14] C. Kittel, Phys. Rev. 70, 965 (1946)

[15] R.E. Rosensweig, Ferrohydrodynamics (Dover Publications, New York, 1985).

[16] G.G. Couto, Nanopartículas de níquel: síntese, caracterização, propriedades e estudo de sua utilização como catalisadores na obtenção de nanotubos de carbono. Dissertação de Mestrado, Universidade Federal do Paraná, Curitiba (2006).

[17] C. Kittel, Phys. Rev. Lett. 73, 810 (1948).

[18] E.S. Leite, Caracterização magnética de óleos magnéticos isolantes para aplicação em transformadores. Dissertação de Mestrado, Universidade de Brasília, Brasília (2009).

[19] C.P. Bean, J. Appl. Phys. 30, 120S (1959).

[20] L.B. Silveira, Estudos de propriedades magnéticas de fluidos e nanocompósitos magnéticos biocompatíveis. Tese de Doutorado, Universidade de Brasília, Brasília (2006).

[21] I.P. Bezerra, Auto-Organização de Aglomerados Finitos de Dipolos Magnéticos Carregados. Dissertação de Mestrado, Universidade Federal do Ceará (2009).

[22] A.F.C. Campos, R. Aquino, F.A. Tourinho, F.L.O. Paula e J. Depeyrot, Eur. Phys. J. E. 36, 42 (2013).

[23] I.T. Lucas, S. Durand-Vidal, O. Bernard, V. Dahirel, E. Dubois, J.F. Dufrêche, S. Gourdin-Bertin, M. Jardat, G. Meriguet e G. Roger, Mol. Phys. 112, 1463 (2014).
[24] C.L. Filomeno, M. Kouyaté, V. Peyre, G. Demouchy, A.F.C. Campos, R. Perzynski, F.A. Tourinho e E. Dubois, J. Phys. Chem. C. 121, 5539 (2017).

[25] R. Messina e H. Löwen, Phys. Rev. Lett. 91, 146101 (2003).

[26] Y. Levin, Rep. Prog. Phys. 65, 1577 (2002).

[27] L. Assoud, R. Messina e H. Löwen, J. Chem. Phys. 129, 164511 (2008).

[28] A.F.C. Campos, F.A. Tourinho, G.J. da Silva, M.C.F.L. Lara e J. Depeyrot, Eur. Phys. J. E 6, 29 (2001).

[29] A. Delattre, S. Pouget, J.F. Jacuot, Y. Samson e P. Reiss, Small 6, 932 (2010).

[30] I.R.O. Ramos, W.P. Ferreira, F.F. Munarin, F.M. Peeters e G.A. Farias, Phys. Rev. E. 85, 051404 (2012). 